\title{
Experimental and numerical investigation of bulging behaviour of hyperelastic textured tubes
}

\author{
Zhiming Guo ${ }^{a}$, Joseph Gattas ${ }^{b}$, Shibin Wang ${ }^{a}$, Linan Li $^{a}$, Faris Albermani ${ }^{b}$ \\ a Department of Mechanics, Tianjin University, China \\ ${ }^{\mathrm{b}}$ School of Civil Engineering, University of Queensland, Australia
}

\begin{abstract}
The inflation and propagation of a localized instability in elastic tubes shares the same mathematical features with a range of other localization problems, including buckling propagation in long metal tubes under external pressure. Recent research into origami-inspired tubular geometries has suggested that geometric texturing is able to significantly improve metal pipe resistance to propagation buckling failures, with an increase in critical and propagation pressures. This paper aims to investigate whether texturing generates a similar improvement in hyperelastic tubes under axial loading and internal pressure, with elastomer origami structures of recent interest for use as soft actuators and robots. A new fabrication method with 3D printed moulds in a dip process was first developed to enable fabrication of textured tube samples. An experimental study was then conducted on inflated smooth and textured latex tubes, with instability formation observed at a $1 \mathrm{~ms}$ resolution. Comparative numerical models with a Mooney-Rivlin material were able to provide a good prediction of experimentally-observed behaviours up to and slightly past the critical pressure and bulge formation. A parametric numerical study is then conducted to show that the number of divisions in the axial direction and circumferential direction have no and modest effects on critical pressure, respectively. The experimental and numerical investigations both showed that the critical pressure of the textured tube was increased compared to the smooth tube, however the degree of increase was a modest $8 \%$ and so unlikely to be of significant practical benefit. This work can provide reference and guidelines for future investigations of tubular inflatable origami structures.
\end{abstract}

\section{Keywords}

Hyperelastic textured tubes; Origami; Finite deformation; Local instability; Inflation

\section{Introduction}

\subsection{Inflation of Elastic Tubes}

Elastic tube inflation shares the same mathematical and mechanical features as a variety of other strain localization phenomena in engineering structures and materials, such as propagating buckling in long metal tubes under external pressure [1], propagating necks [2], stress-induced phase transformation [3], and deformation of aneurysms in human arteries [4]. The inflation of stressed cylindrical tubes is therefore a classic subject of mechanics study, as it provides a good illustration of the phenomenon of initiation and propagation of a localized instability. The instability of rubber tubes and balloons under fluid pressure was first described using a linear material model by Mallock [5]. Subsequent development of finite-elasticity theory [6-8] enabled Kydoniefs and Spencer [9] to obtain the exact solution for an inflated membrane tube using a Mooney-Rivlin model for non-linear rubber material behaviour.

Some studies are devoted to the understanding of tube behaviour under axial loading [10], a description and strain energy function for tube kink-wave type bulging [11], and the bifurcation of the uniform state of thin-walled and thick-walled tubes [12-13]. Chater and Hutchinson [14] presented two examples to illustrate the propagation of bulges and buckles under quasi-static, steady-state conditions, which was used to calculate the propagation pressure of air-inflated rubber tubes [15] and to study the effect of geometric imperfections and material properties on the initiation and propagation of the localized bulging instability [16]. More recent studies of tube instability and localized bulging include application of the Gent rubber model to analyse deformation [17-18], experimental and numerical study of the finite deformation of rubber-like material tubes [19-20], the use of a bifurcation and an energy stability condition to determine the bulge initiation pressure and limit pressure [21], and experimental studies using a high speed camera and data acquisition (DAQ) system [22]. The effect of an initial extension ratio on snap-through instability in tubes was studied in [23], which may occur in elastomeric actuators and artificial muscles. 


\subsection{Origami-Inspired Tubular and Elastomeric Structures}

In recent years origami folding, a traditional artistic form of paper folding, has been of increasing interest to mathematicians and engineers who are finding that traditional geometry and folding can be readily applied to the development of new structures and devices. Numerous works have shown that origami-inspired tubular structures can possess favourable structural behaviours. Origami crash boxes have been shown to have up to a $57 \%$ increase in energy absorption over an equivalent square thin-walled tube [24-25]. Tubular origami for deployable membrane space structures have been investigated and shown to have favourable packaging efficiency and deployment mechanisms [26-27]. Foundational origami science literature proposed the pseudo-cylindrical concave polyhedral (PCCP) shell, which was developed following observations that the post-buckling shape of thin cylindrical shell under axial compression, gave a polyhedral surface that corresponded to a Yoshimura origami pattern [28]. A recent review of further elastic instability and buckling-inspired applications is given in [29]. The PCCP shell was shown to have a higher circumferential bending rigidity compared with a cylindrical shell [28]. This was applied to practical benefit to develop a metal textured subsea pipeline with an improved propagation buckling resistance. Compared to a cylindrical tube, the textured pipeline has a $76 \%$ increase in initiation pressure, a $127 \%$ increase in propagation pressure, and reduced sensitivity to imperfections [30-31].

The majority of origami-inspired engineering research has focused on rigid geometries and materials, which can be folded with minimal deformation of component panels [32-33]. Investigations of elastomeric materials used for origami-inspired applications are relatively few, although increasing because of interest in self-folding and programmable sheets [34]. The potential and limitations of dielectric elastomers for such applications has been investigated [35-36]. Highly stretchable elastomers have been combined with a non-stretchable but easily bendable sheet and shown to be feasible and useful in soft actuators, machines, and robots [37]. In these experiments the elastomer origami actuators show a range of complex motions on pressurization, including extension, contraction, bending, and extension plus torsion.

This study is motivated by the desire to investigate how behaviours of smooth cylindrical inflated hyperelastic tubes compare to origami-inspired textured tube geometries. It is set out as follows. Section 2 first develops a geometric definition and fabrication method for hyperelastic smooth and textured tubes. Section 3 presents an experimental investigation of instabilities in the inflated tubes and a material test for their constituent latex material. Comparative numerical models are then developed in Section 4, with finite element models developed to simulate the localization phenomenon using material constants obtained by nonlinear fitting method. Section 5 presents a discussion of the observed instability behaviour and undertakes a parametric numerical analysis of the textured tube so as to understand which parameters affect critical pressure. The present work is therefore able to act as a reference for the future application of tubular inflatable origami structures.

\section{Geometry and Manufacturing of textured tubes}

\subsection{Geometry of the textured tube}

A parametric definition for a closed, smooth cylindrical tube requires two parameters: centreline radius $R$ and tube length $L$, as shown in Fig. 1a. A textured tube requires a number of extra parameters to be defined, for example side lengths and edge angles. However, as the pattern is a developable and rigid-foldable origami pattern, there is a coupling between these additional parameters and those already defined. Several prior studies have already explored this geometric dependency in depth and this paper shall adopt the parameterisation for an Arc pattern, developed by the second author in [32]. The general form of the Arc pattern, shown in Fig. 1b, requires six independent parameters to be defined. These can be specified from any convenient parameters including volumetric, crease pattern, or angular parameters. The textured tube of interest in the present paper is a restricted configuration of the Arc pattern, in which the pattern is closed and one side length of the component quadrilateral panels is approximately zero, i.e. reduced to triangulated panels as shown in Fig. 1c. A closed textured tube can therefore be defined with four parameters: centreline radius $R$, tube length $L$, the number of divisions along the axial direction $m$, and the number of divisions in the circumferential direction, $n$. The two parameters $m$ and $n$ have been marked in circumferential and axial directions in Fig. $1 \mathrm{~b}$ and 1c, respectively. 


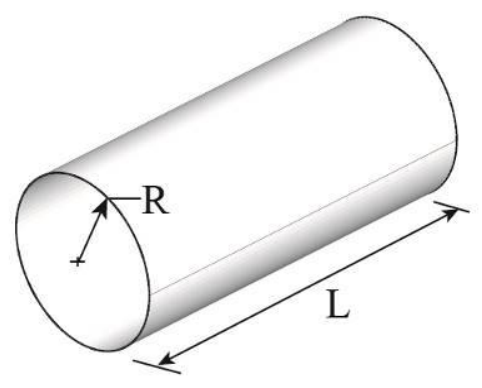

(a)

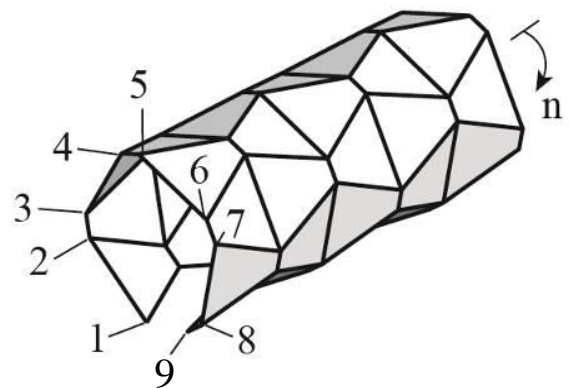

(b)

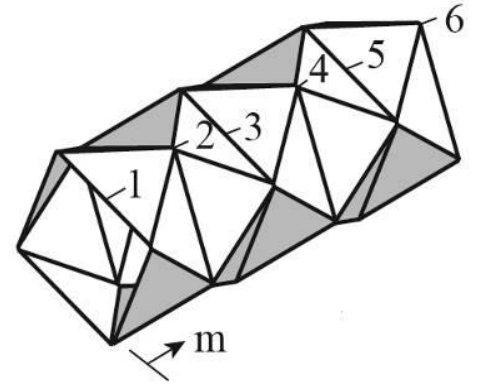

(c)

Fig. 1. Parametric definition of (a) smooth cylindrical tube, (b) general form of Arc pattern, and (c) textured tube.

\subsection{Design and manufacture of hyperelastic textured tubes}

A new manufacturing process was developed in order to fabricate hyperelastic textured tube specimens for subsequent experimental investigation. The design and fabrication process is shown schematically in Fig. 2 and consists of three stages. First, the above parametrisation was implemented in MATLAB and used to generate the textured and smooth tube surfaces. Second, these surfaces were used to create an internal tube mould made of ABS material and 3D printed on the EDEN260V 3D printer from company Objet Geometries. This machine had a $600 \times 600 \times 1600$ dpi print resolution, with printed moulds shown in Fig. 3a. Finally, the moulds were used to make the hyperelastic tubes via a dip process at the Tianjin Rubber Industry Institute. The dip process simply involves dipping the moulds into a latex mixture, drying the coated moulds in an environmentally-controlled chamber, and demoulding, washing, and drying once cured. Samples of the latex material were made with the same process and at the same time.

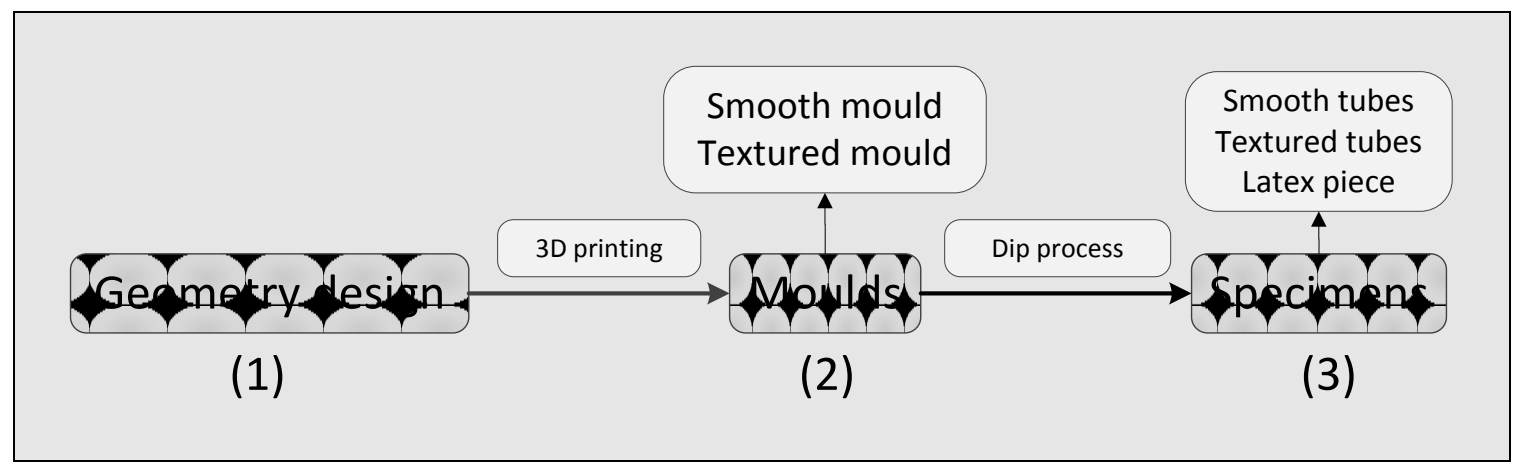

Fig. 2. Manufacturing process of smooth and textured latex tubes.

The manufactured tubes are shown in Fig. $3 b$. Both tubes were specified with $R=5 \mathrm{~mm}$ and $L=200 \mathrm{~mm}(\mathrm{~L} / \mathrm{R}=40)$. The textured tube additionally had $m=40$ and $n=9$. Note that the above geometric parameterisation does not include material thickness, however, the thickness of both physical tubes were measured by thickness gauge to be $0.97 \mathrm{~mm}$ $(R / t=5.15)$. The tube thickness was measured in two directions perpendicular to the tube axis by electronic digital display micrometer, and at three locations along the tube. The variation in thickness between locations was approximately $0.02 \mathrm{~mm}$.
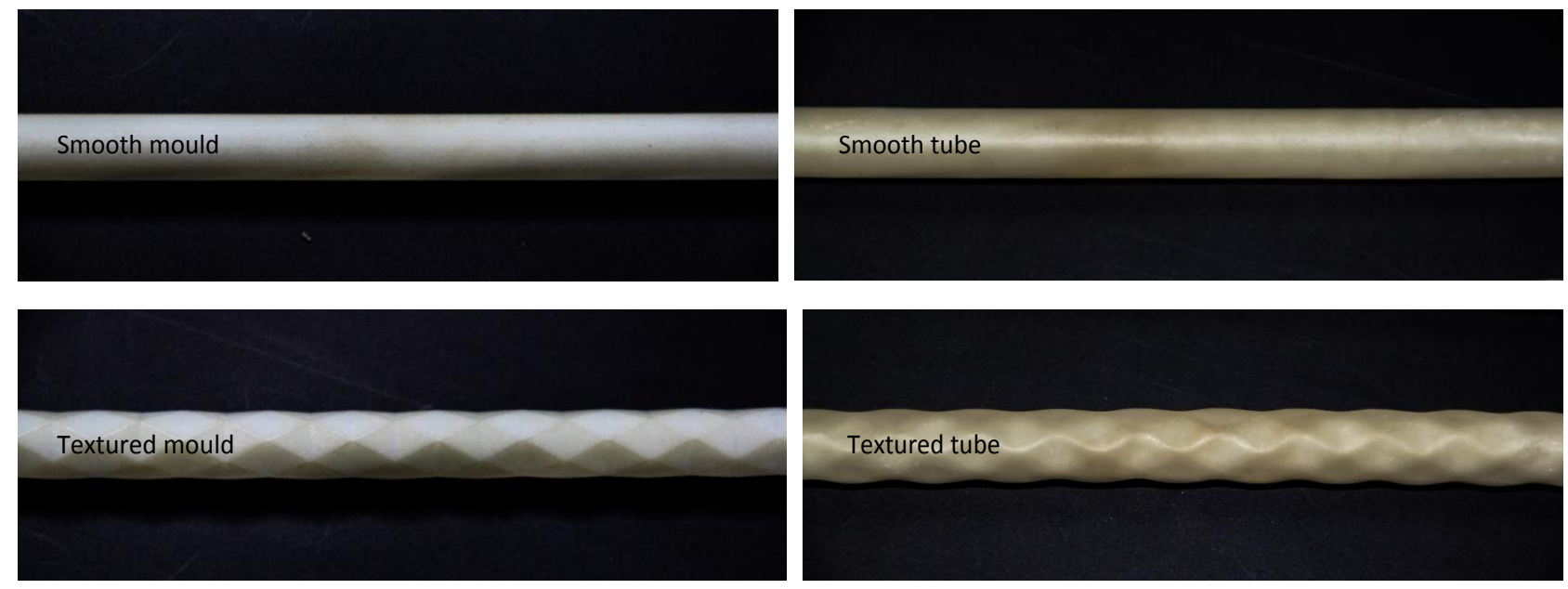
Fig. 3. (a) 3D printed moulds and (b) manufactured smooth and textured latex tubes.

\section{Experimental study}

\subsection{Experimental procedure}

An experimental investigation was conducted to investigate the instability of hyperelastic cylindrical tubes under axial loading and quasi-static inflation of compressed air. The experimental procedure is shown schematically in Fig. 4. Axial load of $1.44 \mathrm{~N}$ was applied with a constant hanging weight. This is followed by inflation of the tube at a constant rate using a pressure regulator and a flow valve. A high frequency pressure sensor was used to measure internal tube pressure, with the transducer output recorded synchronously with the image acquisition system. The image acquisition system includes a high speed camera, a cold light source, and a black backdrop, which enables continuous observation of the tube's configuration as localized deformation initiates and propagates. In this study, the video is recorded at the rate of 1000 frames per second with suitable resolution (192x656 pixels). Finally, the Data Acquisition System was used to collect and store data at the $1 \mathrm{~ms}$ frequency.

The two tubes described in Section 2 were cyclically stretched to achieve the required material stability. The inflation system was then calibrated and used to inflate the two specimens. The inflation rate was controlled by a manual speed regulator and was maintained at a relatively low and constant speed during the experiments. As air was pumped into the tube, a bulge formed at some location along the tube with a corresponding sudden pressure drop at a critical value, $P_{c r}$. With continued inflation, the local bulge grew to a maximum value of radius and then started to gradually propagate along the length while the pressure stayed at a constant value, $P_{p}$. The test was terminated after this constant pressure was reached and before the local bulge reached either end of the specimen.

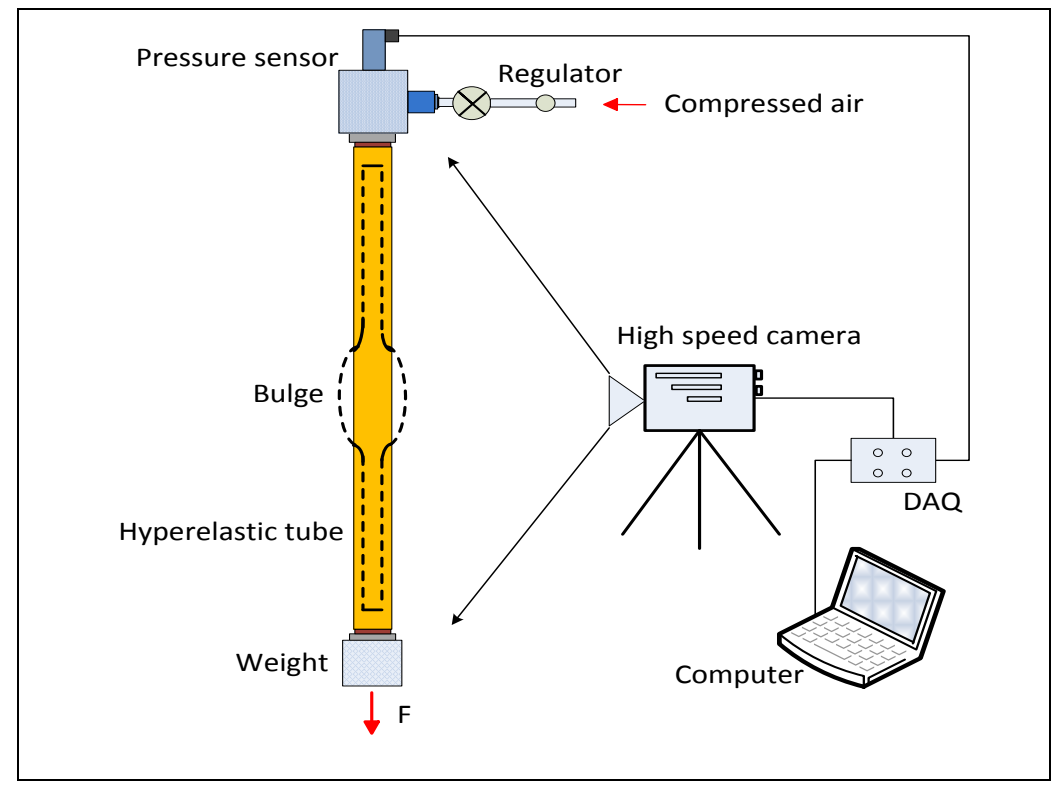

Fig. 4. Schematic diagram of experimental setup.

\subsection{Experimental results}

Bulge formation and propagation occurred in both tube types as shown in Fig. 5. Frame numbers recorded in the figure correspond directly to recorded time shown on the bottom of the figure in milliseconds, with minus frame values due to the method of loop recording in the high speed camera. The position of the bulge was different between tube types, likely due to the effects of material and geometric imperfections. The states of the bulge formation in the smooth tube were consistent with that observed previously, that is the tube diameter increases uniformly until the critical pressure is reached, after which the diameter continues to grow at the bulge location but reduces elsewhere along the tube. Observation of the states of the textured tube shows that it follows the same process as the smooth tube. This is considered in depth as follows. 


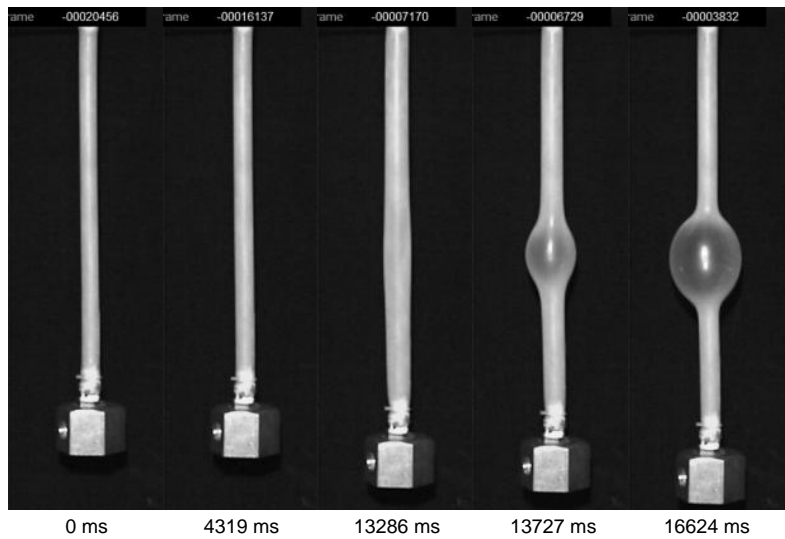

(1)

(2)

(3)

(4)

(5)

(a)

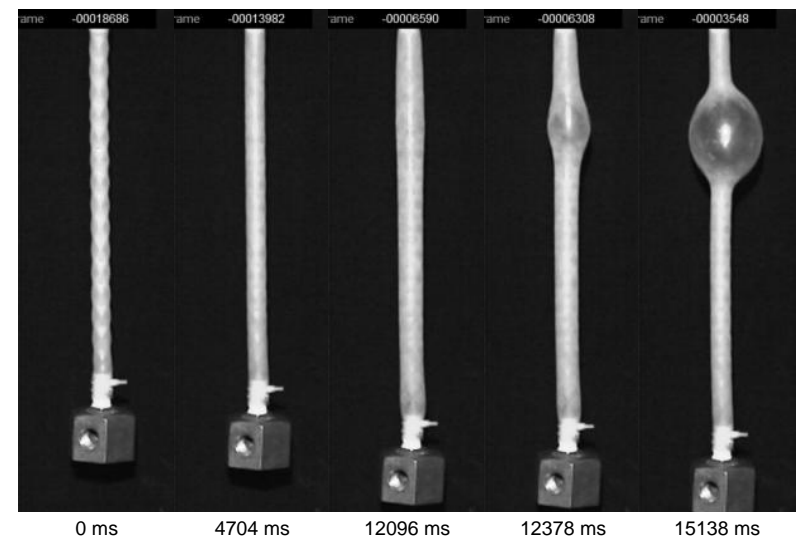

(1)

(2)

(3)

(4)

(5)

(b)

Fig. 5. Bulge formation of the inflated tubes in the presence of axial loading. (a) Smooth tubes and (b) textured tubes.

An image of the localized instability obtained by the high speed camera is shown in Fig. 6 . The bulge is assumed to be symmetric and the location along the tube axis at the widest point of the bulge is termed point $O$. The initial diameter of the tube is $D=2 R=10 \mathrm{~mm}$ and the diameter of bulge at point $\mathrm{O}$ is defined as $d$ which is shown in Fig. 6 . The radial displacement of the bulge then defined as Dis and calculated as $\left(d-D_{\text {initial }}\right) / 2$, where $D_{\text {initial }}$ is diameter of the tube after axial load is applied but prior to inflation. The high contrast between the white tube and the black background enabled MATLAB's image processing tools to be used to quantify the radial displacement for every captured image, i.e. in $1 \mathrm{~ms}$ increments. For processing, each row of pixels was scanned to obtain a first and last 'white' pixel location. The largest difference between pixel locations was taken as the bulge diameter and converted from pixels to $\mathrm{mm}$ by using a conversion factor calculated from the known width of the suspended weight at the bottom end of the tube specimens. This method was also used to calculate $D_{\text {initial }}$ as $9.68 \mathrm{~mm}$.

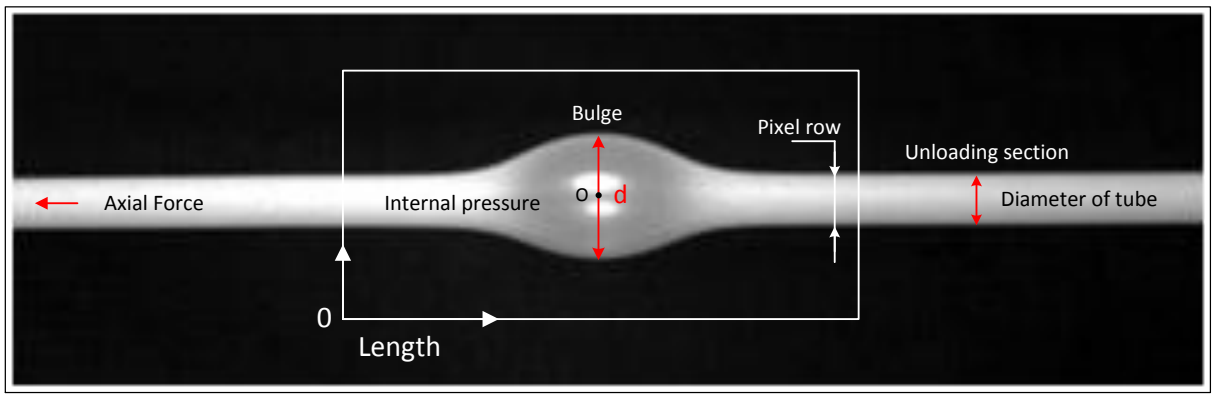

Fig. 6. Loading diagram and deformation of the bulge.

The measured pressure and displacement values are plotted against time in Fig. 7, with the entire test period shown in Fig. $7 \mathrm{a}$ and the local 200ms time period for bulge formation shown in Fig. $7 \mathrm{~b}$. Nine sequential images of the bulge formation and corresponding bulge profiles are shown in Fig. 8. They correspond to 20ms increments denoted with blue star marks in Fig. 7. The critical pressure was found to be $0.0625 \mathrm{MPa}$ at time $14989 \mathrm{~ms}$. The bulging period then followed from $15420 \mathrm{~ms}$ to $15580 \mathrm{~ms}$, with the diameter of the tube increasing by $156.6 \%$ at the bulge and decreasing by $41.9 \%$ in the section away from the bulge. Fig. $8 \mathrm{~b}$ shows that the bulging speed, i.e. rate of radial displacement is nonlinear, and that the sequential profiles all intersect at one point. This implies that there is a common solution during formation of the bulge. 


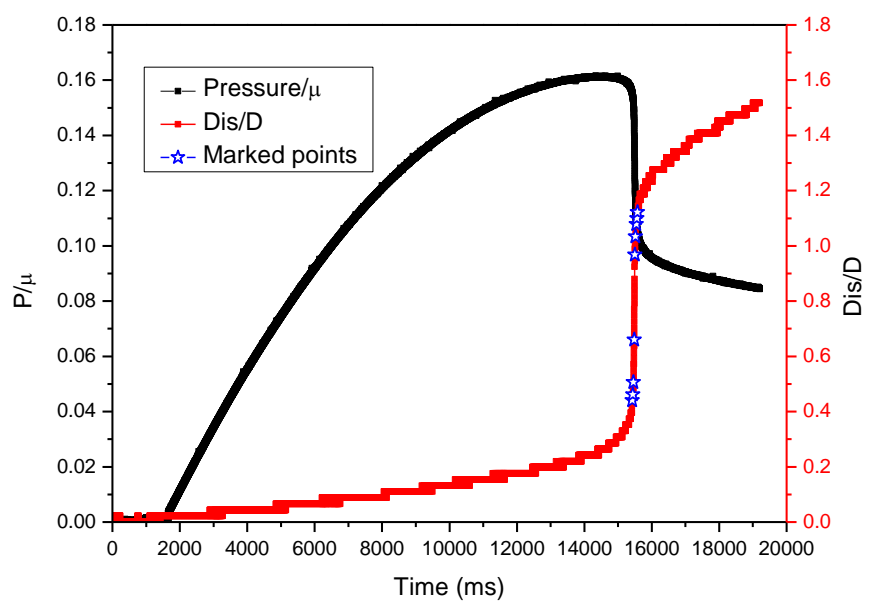

(a)

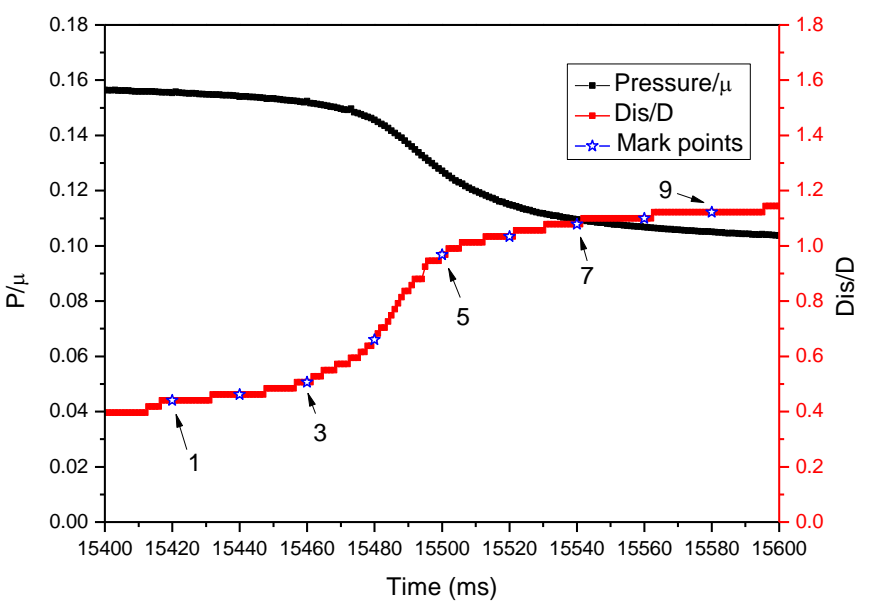

(b)

Fig. 7. Pressure-time history of the smooth tube. (a) The entire test and (b) the region of the instability.

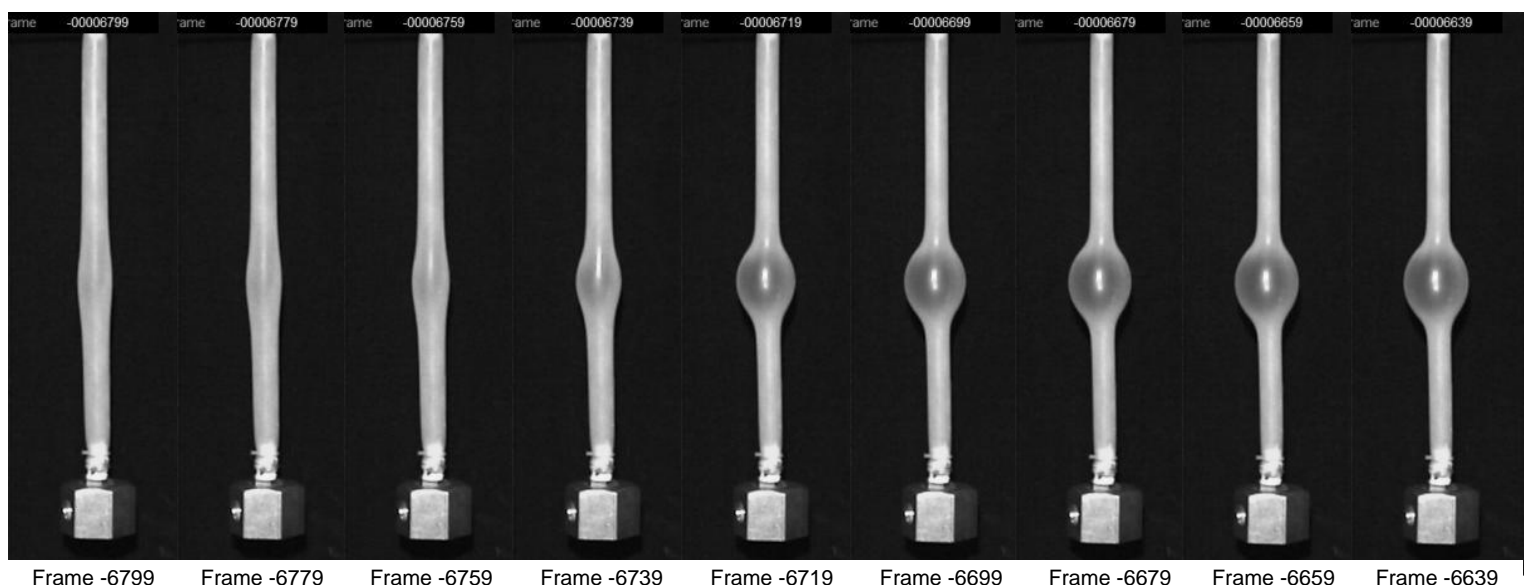

(1)

(2)

(3)

(4)

(5)

(6)

(7)

(8)

(9)

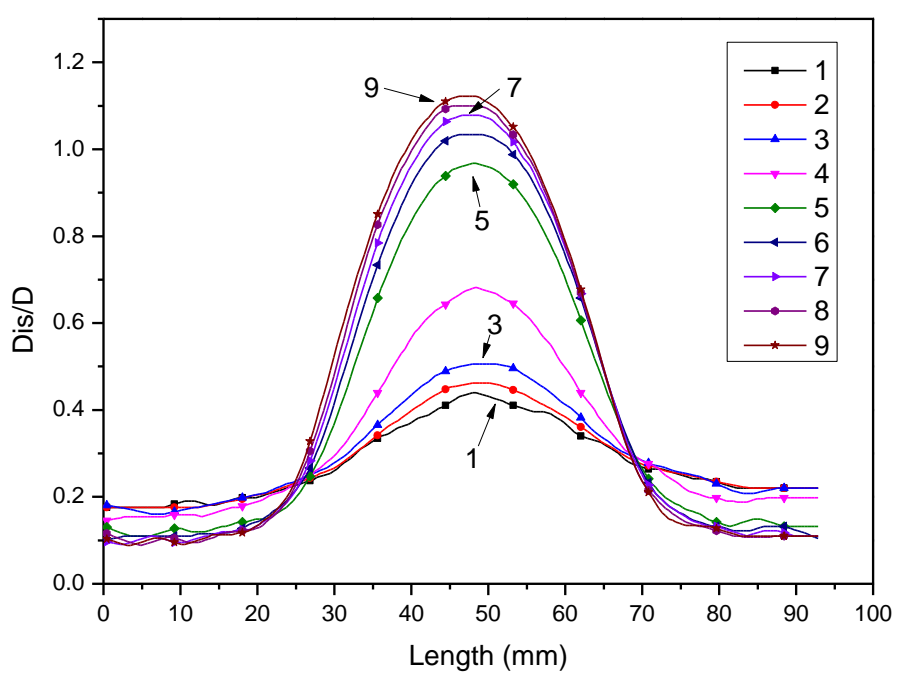

Fig. 8. Experimental smooth tube bulge configurations (top) and profiles (bottom) under different pressures.

A similar image processing was conducted on the textured tube sample, with results shown in Fig. 9 and Fig. 10. The critical pressure was found to be $0.0679 \mathrm{MPa}$ at time $13077 \mathrm{~ms}$. The bulge formation follows in the period of 13420 $\mathrm{ms}$ to $13580 \mathrm{~ms}$, with the diameter of the tube increasing by $180 \%$ at the bulge location and decreasing by $49.6 \%$ in the section away from the bulge. The initial volume of the textured tube is slightly less than that of the cylindrical tube, hence under similar inflation rate for both, less time is needed to reach initiation in the textured tube. Fig. 10 shows sequential images and bulge profiles at $20 \mathrm{~ms}$ increments corresponding to blue points marked on Fig. 9. The nonlinear bulging speed is again exhibited along with sequential bulge profiles with a single common intersection point. The pressure and radial displacement relationship for both tubes are plotted in Fig. 11. Note that the pixelated 
appearance of the plot is due to the pixel measure method used to obtain displacement, rather than from graph quality.

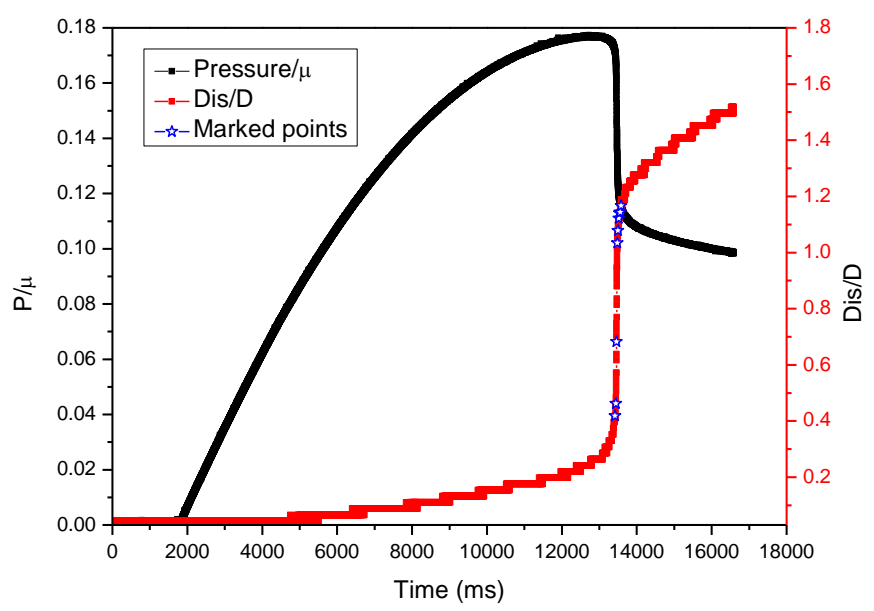

(a)

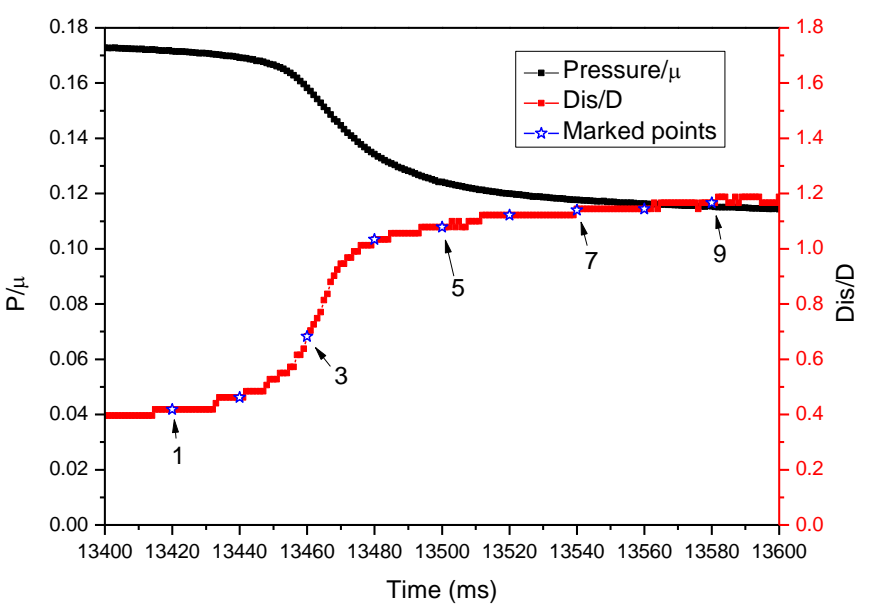

(b)

Fig. 9. Pressure-time history of the textured tube. (a) The entire test and (b) the region of the instability.

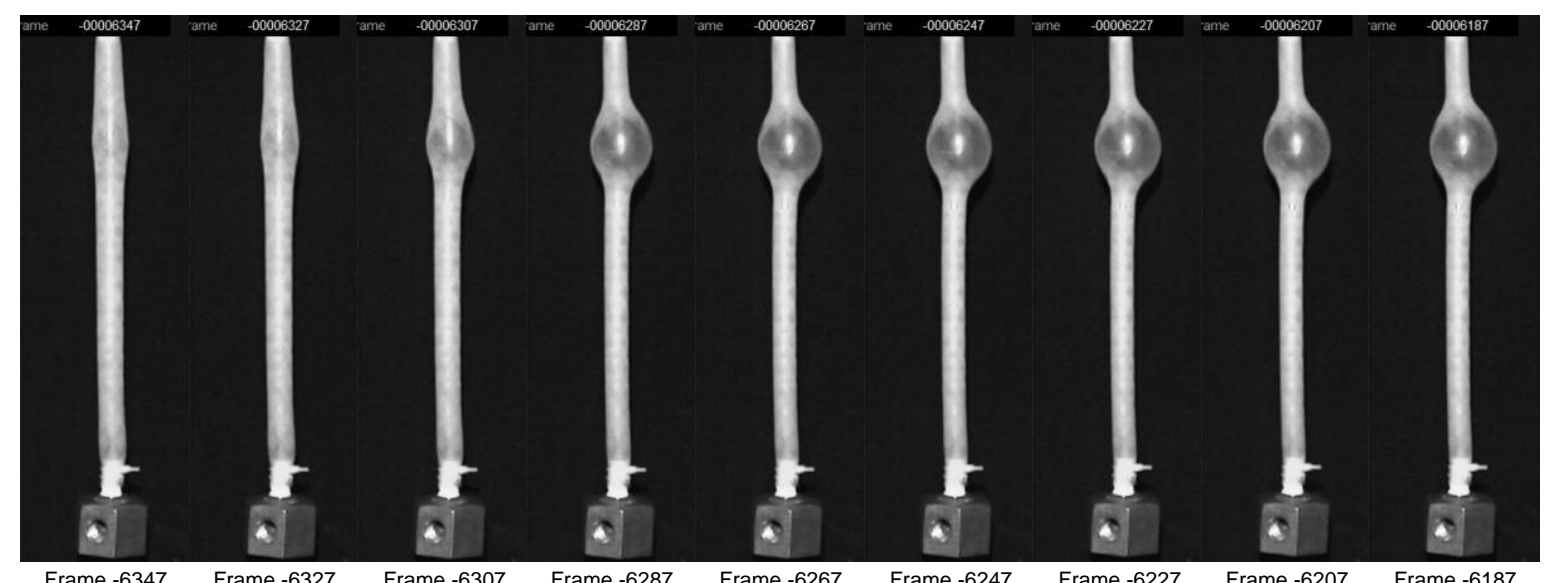

(1)

(2)

(3)

(4)

(5)

(6)

(7)

(8)

(9)

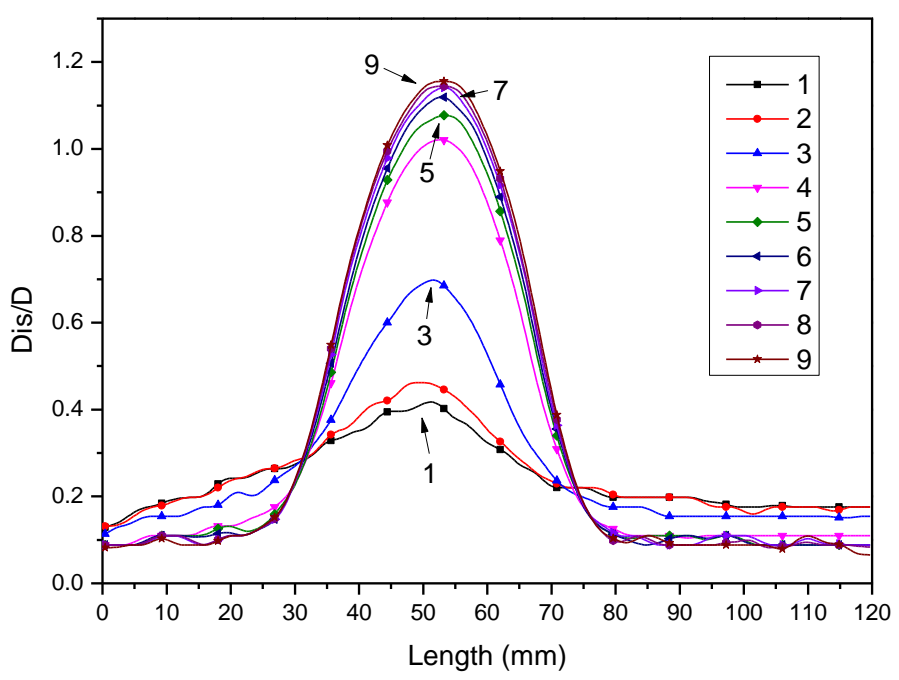


Fig. 10. Experimental textured tube bulge configurations (top) and profiles (bottom) under different pressures.

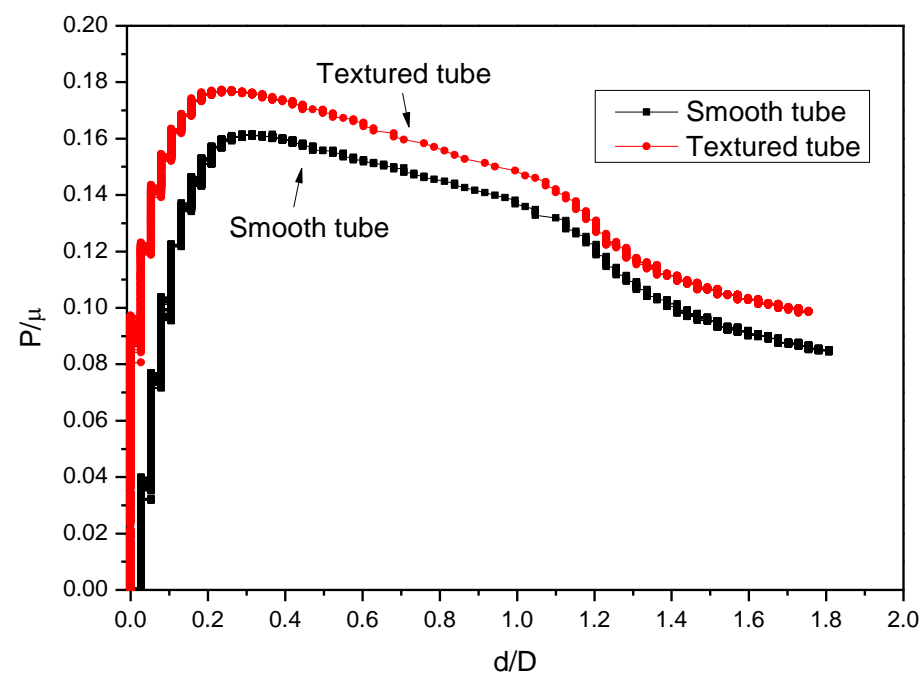

Fig. 11. Measured experimental normalized pressure-displacement curves of smooth and textured tubes.

\subsection{Material tensile tests}

A flat piece of latex was made with the same process and at the same time as the tube samples. One of the dumbbell-shaped latex specimens, shown in Fig. 12a, was cut from this sheet and tested under tensile loading as shown in Fig. 12c. Samples were marked with two black dots at a spacing of $25 \mathrm{~mm}$. The stretch ratio was measured with a non-contact method and the $\sigma_{E} \sim \lambda$ curves were obtained as shown in Fig. 14a. In these curves, $\sigma_{E}$ is $F / A$, where $F$ is axial loading and $A$ was the initial cross sectional area. The extension ratio is $\lambda=(L+\Delta L) / L$, where $\Delta L$ is the change in length and $L$ is the initial length. Five samples were tested and the average $\sigma_{E} \sim \lambda$ curve is obtained as shown in Fig. 14a.

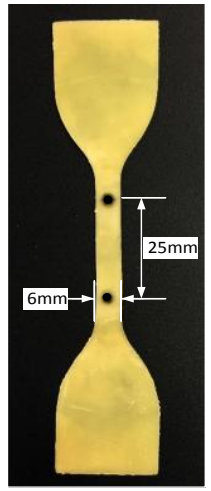

(a)

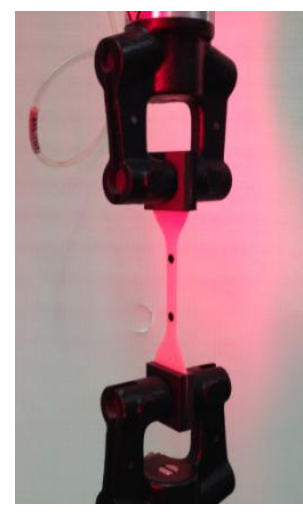

(b)

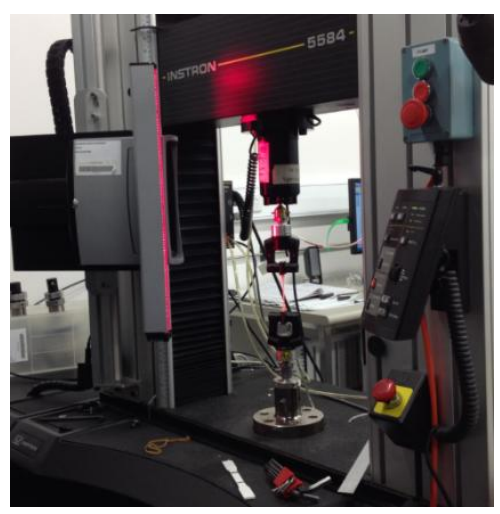

(c)

Fig. 12. Latex material tests. (a) Dumbbell-shaped specimen marked with dots, (b) pneumatic fixture used to clamp sample, and (c) tensile test.

\section{Numerical analysis}

\subsection{Finite element models}

A comparative numerical model was developed to simulate the behaviour observed in experiments and understand the nonlinear behaviour of the smooth and textured tubes. Finite element models were thus developed with commercial software ABAQUS. Models used S4R shell elements for discretization and a Riks solution method. Material properties are as discussed in the next section. Boundary conditions were applied at both ends of the tube. At one end, all nodal displacements were fixed. At the other end, all nodal displacements except for the axial degree of freedom were fixed. A constant weight $(1.44 \mathrm{~N})$ was maintained at this end before and during inflation. A meshsensitivity analysis was conducted to obtain a converged mesh size with 6138 nodes and 6107 elements in smooth model, and 7992 nodes and 8424 elements in the textured model. The two models are shown in Fig. 13, with the same geometric features as discussed previously: initial length $L=200 \mathrm{~mm}$, initial thickness $t=0.97 \mathrm{~mm}$, initial radius $\mathrm{R}=5 \mathrm{~mm}$ and for the textured tube $\mathrm{m}=40$ and $\mathrm{n}=9$. 


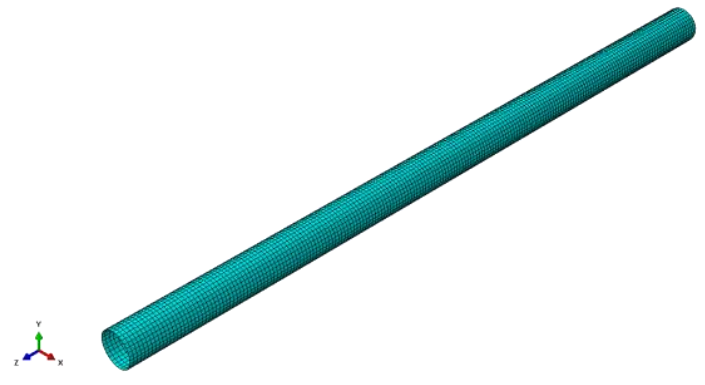

(a)

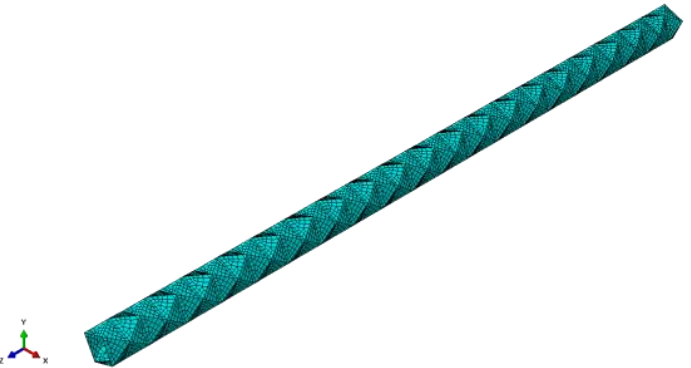

(b)

Fig. 13. Finite element models of hyperelastic (a) smooth and (b) textured tubes.

\subsection{Material properties}

As discussed in Section 2, both tubes were manufactured using a dipping process with commercial natural latex. This material was assumed to be isotropic, homogeneous, incompressible and hyperelastic. According to the principle of complete recoverability after deformation, the strain energy density function can be described by the principal stretches $\lambda_{1}, \lambda_{2}$ and $\lambda_{3}$ or by the strain invariants $I_{1}, I_{2}$ and $I_{3}$, which is

$W=W\left(\lambda_{1}, \lambda_{2}, \lambda_{3}\right)=W\left(I_{1}, I_{2}, I_{3}\right)$

where $W$ is the strain-energy function that is usually used to describe the hyperelastic material. The relationship between the principal stretches and the strain invariants is given by

$$
I_{1}=\lambda_{1}^{2}+\lambda_{2}^{2}+\lambda_{3}^{2}, I_{2}=\left(\lambda_{1} \lambda_{2}\right)^{2}+\left(\lambda_{2} \lambda_{3}\right)^{2}+\left(\lambda_{1} \lambda_{3}\right)^{2}, I_{3}=\left(\lambda_{1} \lambda_{2} \lambda_{3}\right)^{2}
$$

Because of the very small volume changes of rubber-like materials, the incompressibility is usually taken into consideration, so we obtain $\lambda_{1} \lambda_{2} \lambda_{3}=1$.

There are several models developed to describe the mechanical behaviour of elastomers and soft tissues under large strains such as Neo-Hookean [38], Mooney-Rivlin [39], Ogden [40], and Gent [18]. We will select the model proposed by Mooney and Rivlin as it is widely used in numerical analysis and in the studies of finite deformation of hyperelastic materials. The Mooney-Rivlin model contains two material constants $C_{1}$ and $C_{2}$ that are obtained from the material stress-strain behaviour.

$W=C_{1}\left(I_{1}-3\right)+C_{2}\left(I_{2}-3\right)$

The relationship between the engineering stress $\sigma_{E}$ and extension ratio $\lambda$ in the tensile direction can be derived as

$\sigma_{E}=\frac{2\left(C_{2}+\lambda C_{1}\right)\left(\lambda^{3}-1\right)}{\lambda^{3}}$
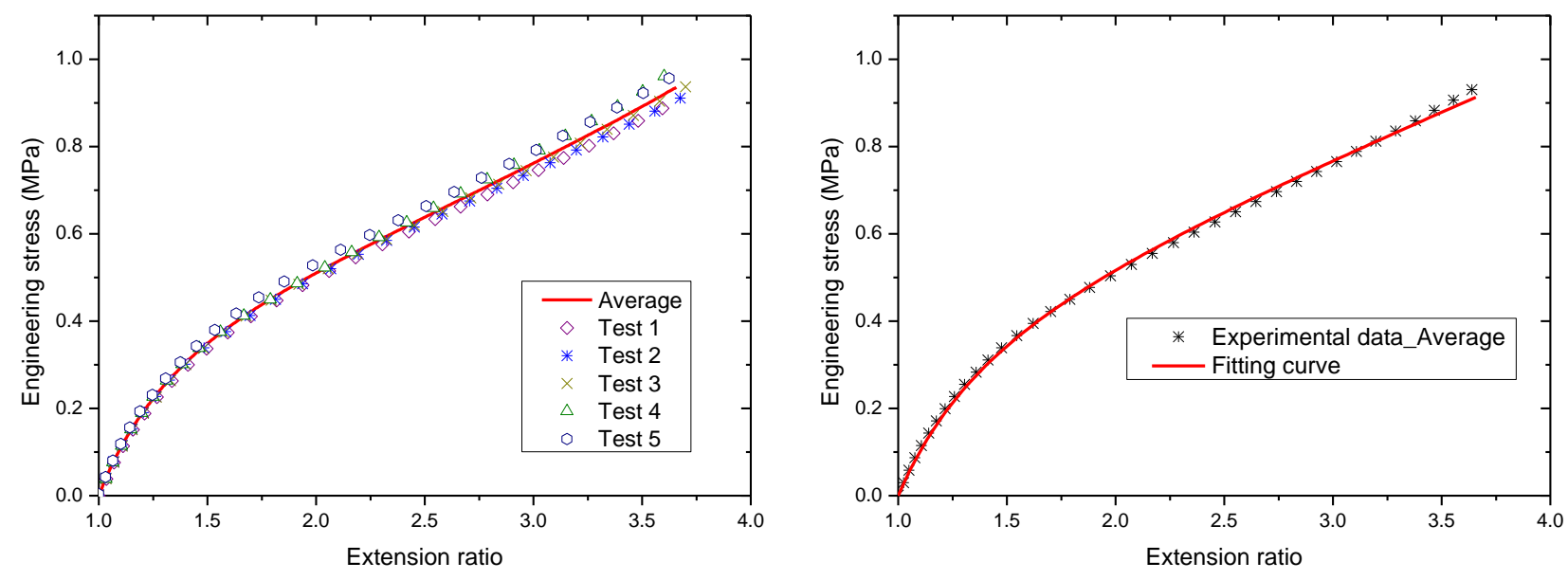
Fig. 14. (a) Material testing results of axial load as a function of extension ratio. (b) Material constants obtained by nonlinear fitting method.

The material constants were fitted with the average data from the material test experiments, shown in Fig. 14a, and the theoretical relation (4). The fitted curve is shown in Fig. 14b, with the strain energy function (3) and nonlinear fitting method was used to obtain material parameters. The constants are obtained as $C_{1}=0.10313 \mathrm{MPa}, C_{2}=$ $0.08878 \mathrm{MPa}$, and shear modulus $\mu$ is $2\left(C_{1}+C_{2}\right)$.

\subsection{Numerical simulation of experimental tubes}

Shown in Fig. 15 is the relationship between internal pressure and the maximum radial displacement as obtained from numerical models. Numerical models both exhibit the bulge formation and propagation as expected, with critical pressures of $0.0615 \mathrm{MPa}$ and $0.0667 \mathrm{MPa}$ for smooth and textured tubes, respectively. The stages of bulge formation and measured Von Mises stress are shown in Fig. 16. During the uniform deformations from configuration (1) to (2), the stress distribution is uniform in the smooth tube but non-uniform in the textured tube.

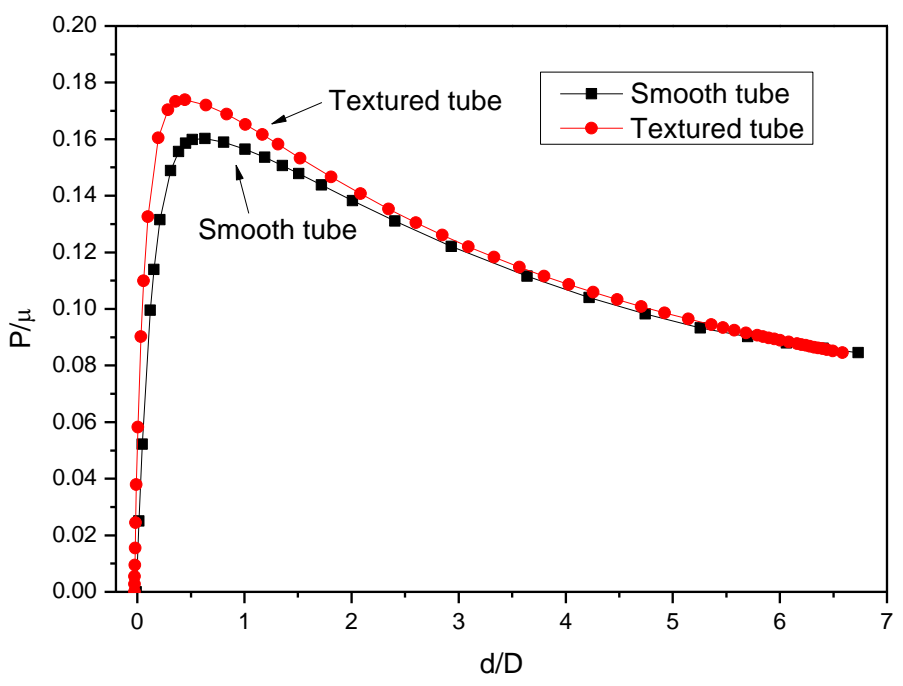

Fig. 15. The normalized pressure-displacement curves of numerical smooth and textured tubes.

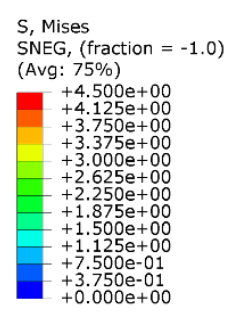

(a)

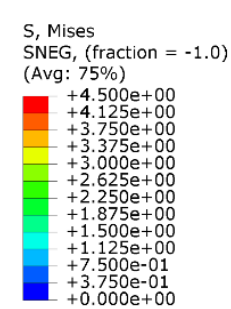

(b)

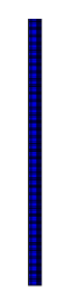

(1)

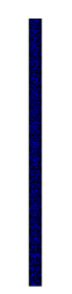

(1)

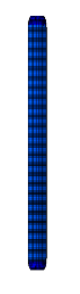

(2)

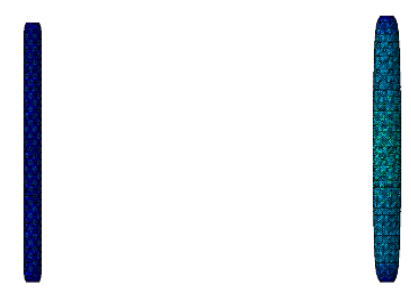

(2)

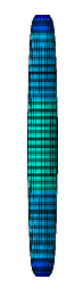

(3)

(3)

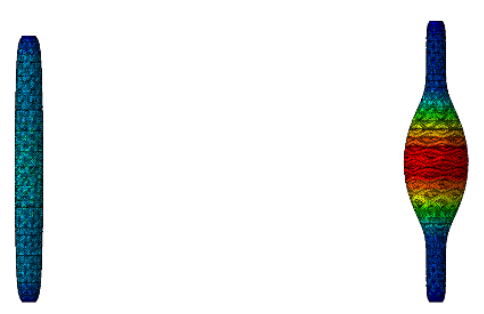

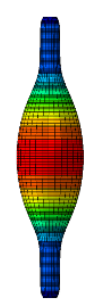

(4)

(4)

Fig. 16. Sequence of deformed configurations for (a) smooth tube and (b) textured tube.

\section{Discussion}

\subsection{Comparison of numerical and experimental results}

The experimental and numerical results show that the localization occurred in both types of hyperelastic tubes. With inflation, the internal pressure reaches a limit point and the tube stiffness decreases, after which the internal pressure decreases sharply as the bulging forms and the diameter of unloading section decreases. Values from numerical and experimental results are summarised in Table 1 and Fig. 17a and b. There is good agreement for both 
critical pressure and the corresponding maximum change of radial displacement until the onset of bulging, with the difference between experimental and numerical critical pressures as $1.6 \%$ for smooth tube, and $1.8 \%$ for textured tube. The critical pressure of the textured tube is seen to be higher than the smooth tube, but only by a modest $8 \%$.

This a much smaller effect to that observed previously for comparisons of elastic-plastic smooth and textured tubes [30-31]. This can be understood with observation of the central cross section configurations of smooth and textured tubes, shown in Fig. 17c and d. Initial and critical configurations correspond to the indicated pressure points in Fig. 17a. The reduced effect of the textured tube is attributed to the large radial deformation that the hyperelastic textured tube undergoes prior to instability formation. This acts to reduce the dihedral angles $\theta$ of adjacent plates and thus 'smooths' the textured tube shown in Fig. 17d. This differs from results previously reported for the improved buckling response of metallic textured tubes [31], as dihedral angles were present during instability formation, which caused an increased second moment of area and shell stiffness, and thus an increased critical pressure. It can also be seen that textured effect on stress development prior bulge formation is small.

Finally, a large numerical-experimental discrepancy is seen in the equilibrium paths occurring after instability formation, with numerical models undergoing much larger displacements. This is likely due to assumptions in the material model. It is probable that the Mooney-Rivlin material model is not quite suitable for modeling bulge propagation, since that the pressure versus volume curve does not have a minimum, and so a Maxwell state corresponding to steady propagation cannot be reached [41].

Table 1

Comparison between experimental and numerical results of critical pressure

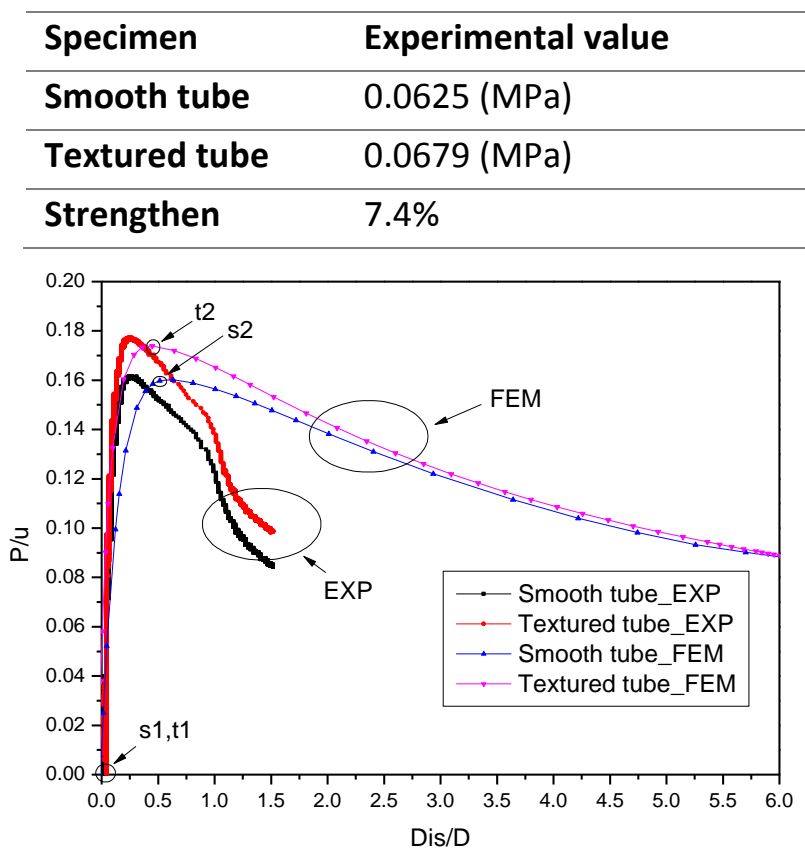

(a)

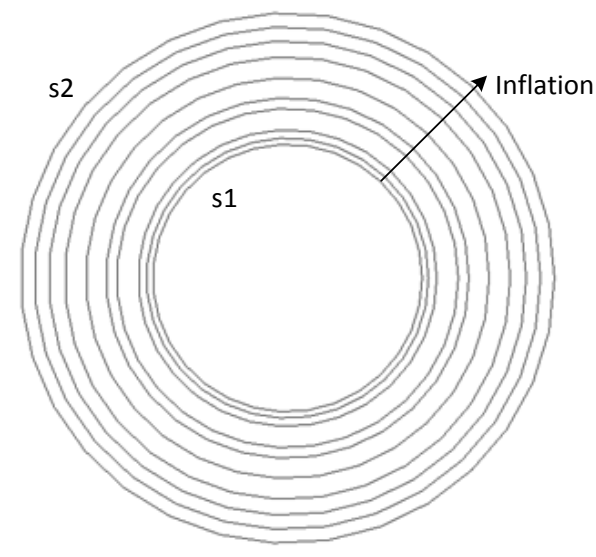

(c)

\begin{tabular}{ll} 
Numerical value & Difference \\
\hline $0.0615(\mathrm{MPa})$ & $1.6 \%$ \\
$0.0667(\mathrm{MPa})$ & $1.8 \%$ \\
$8.5 \%$ &
\end{tabular}

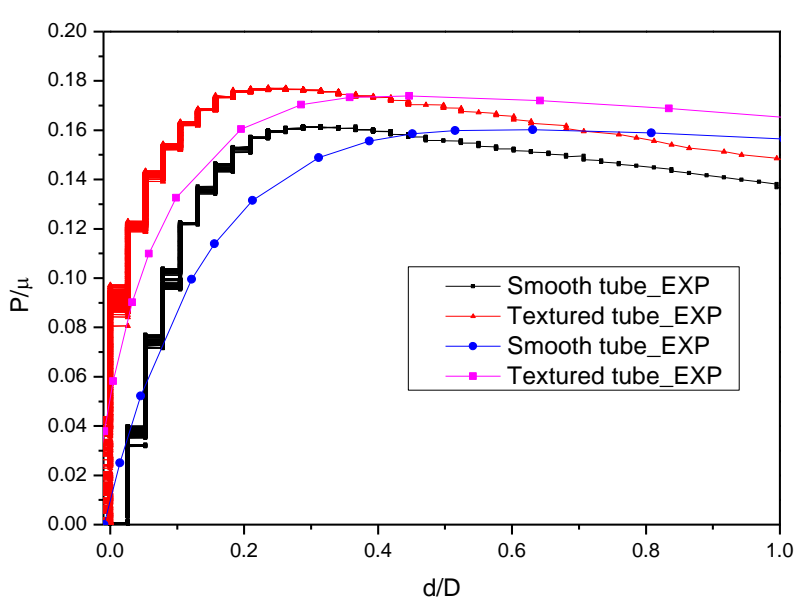

(b)

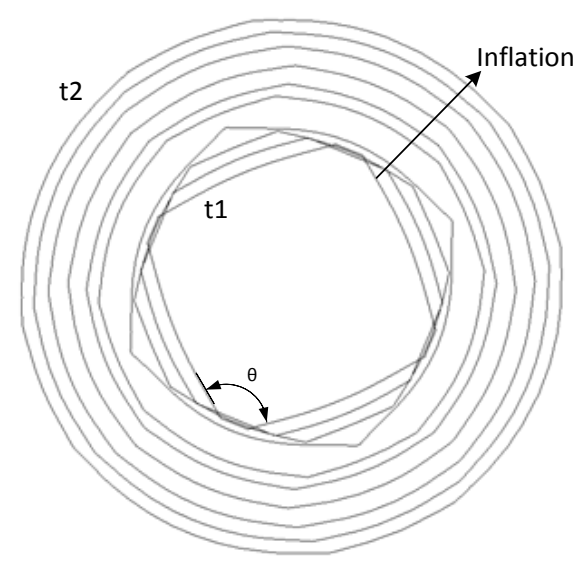

(d) 
Fig. 17. Comparison of experimental and numerical pressure-displacement curves of smooth/textured tubes. (a) The entire test and (b) the region of the instability, and (c) the numerical configurations at center section of smooth tube, and of textured tube (d).

\subsection{Parametric numerical analysis of textured tubes}

An aspect of tube performance that could not be explored experimentally was the effect of additional textured tube parameters $m$ and $n$. As the numerical model for the textured tube was seen to provide good prediction of the critical pressures observed in experimental models, a parametric numerical study was conducted to see how this was affected by these two parameters.

The effect of parameter $n$ was first studied with eight numerical models with values of $n$ between 7 and 29. Other parameters were the same as used previously. Values are summarised in Table 2 and it can be seen that the instability is still observed in all tubes, with critical pressure decreasing with the increase of $\mathrm{n}$. It is noted that there is a critical $n$ value $(n=17)$ at which the textured tube has an approximately equivalent critical pressure to the smooth tube $(0.0615 \mathrm{MPa})$. Below this point, the reduction in $\mathrm{n}$ increases the degree of texturing present in the tube, which acts to enhance the critical pressure since the second moment of area and circumferential bending stiffness of the tube was changed, consistent with that observed in [31]. Above this point, the increase in $n$ generates a large number of the ridgelines between facets which act as an imperfection to lower the critical loading below that observed in the smooth tube. Since localized bulging is a subcritical bifurcation phenomenon [21], these imperfections can lower the initiation pressure below that of the smooth tube, and so numerical results are consistent with that reported in [42].

Table 2

Parameter $\mathrm{n}$ and corresponding critical pressure

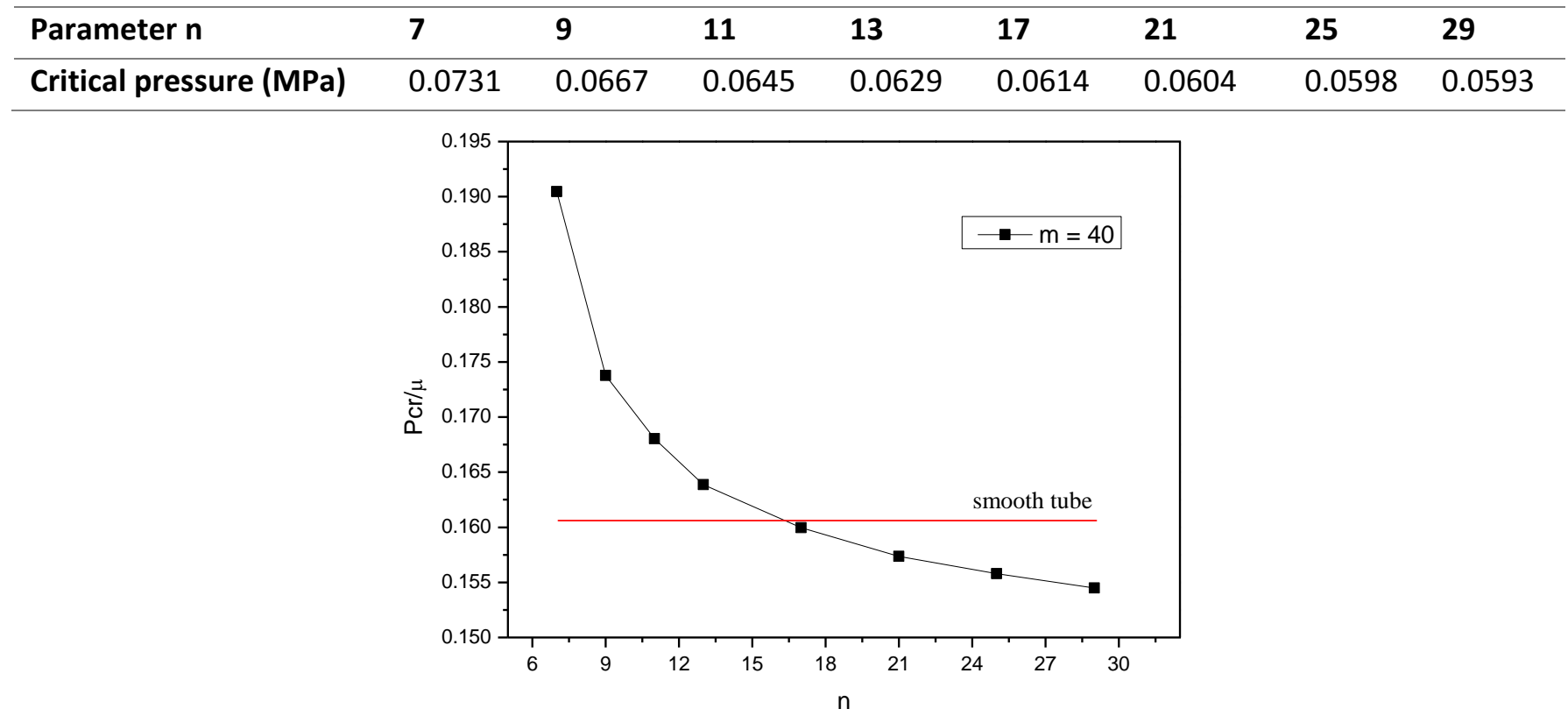

Fig. 18. Influence of the parameter $n$ on the critical pressure of textured tube.

The effect of parameter $m$ was also studied with six models with values of $m$ between 10 and 60 . Other parameters were held constant. Critical pressure values are summarised in Table 3 and results plotted in Fig. 19. It can be seen that the critical pressure remains almost constant and so the effect of parameter $m$ on the instability is minimal. This is attributed to the initial axial loading causing an extension in the tube and corresponding reduction in the degree of texturing, i.e. a smoothing, between adjacent panels.

Table 3

Parameter $\mathrm{m}$ and corresponding critical pressure

\begin{tabular}{lllllll}
\hline Parameter $\mathbf{m}$ & $\mathbf{1 0}$ & $\mathbf{2 0}$ & $\mathbf{3 0}$ & $\mathbf{4 0}$ & $\mathbf{5 0}$ & $\mathbf{6 0}$ \\
\hline Critical pressure (MPa) & 0.062853 & 0.06290 & 0.06290 & 0.06288 & 0.06298 & 0.06305 \\
\hline
\end{tabular}




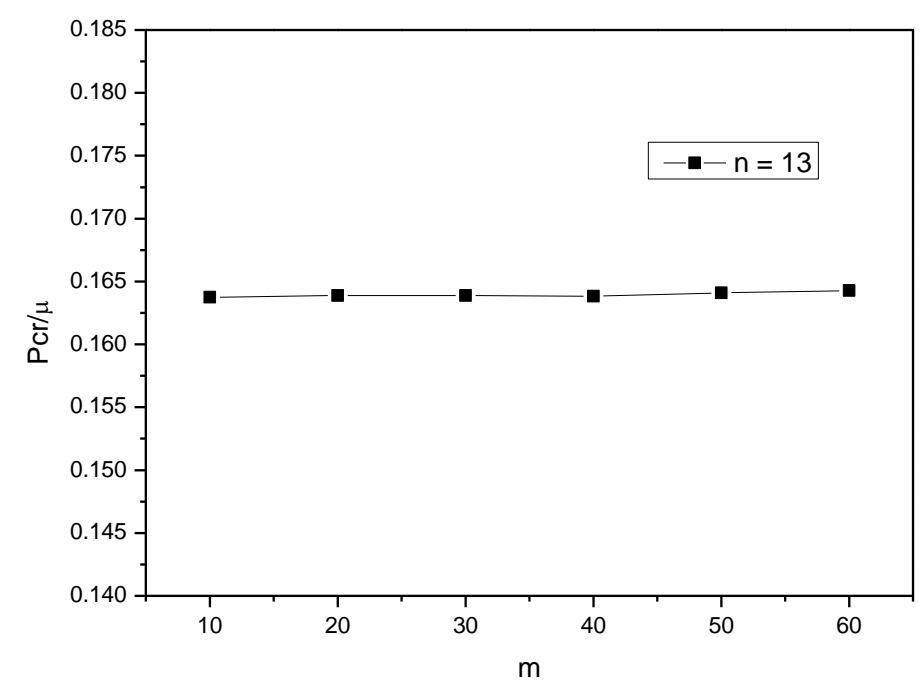

Fig. 19. Influence of the parameter $m$ on the critical pressure of textured tube.

\section{Conclusion}

This paper has studied the nonlinear behaviour of cylindrical hyperelastic tubes that have smooth and origamiinspired textured structures. It is the first investigation of this kind and has demonstrated several key findings of benefit in future development of tubular inflatable origami structures. First, it was shown that effect of the textured geometry on critical pressure was limited, having only an $8 \%$ improvement compared to a smooth tube. This behaviour in hyperelastic material instabilities is different to that previously reported for instabilities in elastic-plastic textured tubes and was attributed to the large radial displacement undergone by textured hyperelastic tubes prior to instability formation. Second, for the selected tubular geometry, it was shown that the parameter $\mathrm{n}$ plays an important role on instability but $m$ has little impact on critical pressure. Finally, a novel manufacturing method using 3D printed moulds and a dipping process was shown to enable straightforward manufacture of textured tubes and thus the analyses conducted in the paper. This can be applied to other tubular geometries for investigation of potential novel mechanical properties, for example further instability behaviours or deployment mechanisms in fields such as soft robotics.

\section{Acknowledgement}

This work was supported by the National Science Foundation of China (Grant No. 11372212). The first author Zhiming Guo expresses his sincere gratitude to the China Scholarship Council for financial support to study at the University of Queensland (CSC NO. 201406250098). We would like to acknowledge Prof. Yibin Fu of Keele University for his help and advice on this research work.

\section{References}

[1] Kyriakides S, Babcock CD. Experimental determination of the propagation pressure of circular pipes. Journal of Pressure Vessel Technology 1981;103:328-336.

[2] Hutchinson JW, Neale KW. Neck propagation. J Mech Phys Solids 1983;31:405-426.

[3] Ericksen JL. Equilibrium of bars. J. Elast. 1975;5:191-201.

[4] Fu YB, Rogerson GA, Zhang YT. Initiation of aneurysms as a mechanical bifurcation phenomenon. Int J Non Linear Mech 2012;47:179-184.

[5] Mallock A. Note on the instability of India-rubber tubes and balloons when distended by fluid pressure. Proceedings of the Royal Society of London 1890;49:458-463.

[6] Rivlin RS. Large elastic deformations of isotropic materials. I. Fundamental concepts. Philosophical Transactions of the Royal Society of London. Series A, Mathematical and Physical Sciences 1948;240:459-490.

[7] Rivlin RS. Large elastic deformations of isotropic materials. III. Some simple problems in cylindrical polar coordinates. Philosophical Transactions of the Royal Society of London A: Mathematical, Physical and Engineering Sciences 1948;240:509-525.

[8] Green AE, Adkins JE. Large elastic deformations and non-linear continuum mechanics, Oxford: Clarendon Press, 1960. 
[9] Kydoniefs AD, Spencer AJM. Finite axisymmetric deformations of an initially cylindrical elastic membrane. The Quarterly Journal of Mechanics and Applied Mathematics 1969;22:87-95.

[10] Alexander $\mathrm{H}$. The tensile instability of an inflated cylindrical membrane as affected by an axial load. Int. J. Mech. Sci. 1971;13:87-95.

[11] Yin WL. Non-uniform inflation of a cylindrical elastic membrane and direct determination of the strain energy function. J. Elast. 1977;7:265-282.

[12] Haughton DM, Ogden RW. Bifurcation of inflated circular cylinders of elastic material under axial loading-I. Membrane theory for thin-walled tubes. J Mech Phys Solids 1979;27:179-212.

[13] Haughton DM, Ogden RW. Bifurcation of inflated circular cylinders of elastic material under axial loading-II. Exact theory for thick-walled tubes. J Mech Phys Solids 1979;27:489-512.

[14] Chater E, Hutchinson JW. On the propagation of bulges and buckles. Journal of Applied Mechanics 1984;51:269277.

[15] Kyriakides S, Yu-Chung C. On the inflation of a long elastic tube in the presence of axial load. International journal of solids and structures 1990;26:975-991.

[16] Kyriakides S, Yu-Chung C. The initiation and propagation of a localized instability in an inflated elastic tube. International Journal of Solids and Structures 1991;27:1085-1111.

[17] Gent AN. Elastic instabilities in rubber. Int J Non Linear Mech 2005;40:165-175.

[18] Gent AN. A new constitutive relation for rubber. Rubber chemistry and technology 1996;69:59-61.

[19] Pamplona DC, Goncalves PB, Lopes SRX. Finite deformations of cylindrical membrane under internal pressure. Int. J. Mech. Sci. 2006;48:683-696.

[20] Goncalves PB, Pamplona D, Lopes SRX. Finite deformations of an initially stressed cylindrical shell under internal pressure. Int. J. Mech. Sci. 2008;50:92-103.

[21] Fu YB, Pearce SP, Liu KK. Post-bifurcation analysis of a thin-walled hyperelastic tube under inflation. Int J Non Linear Mech 2008;43:697-706.

[22] Guo Z, Wang S, Li L, Ji H, Wang Z, Cai S. Inflation of stressed cylindrical tubes: an experimental study, in: International Conference on Experimental Mechanics 2013 and the Twelfth Asian Conference on Experimental Mechanics, International Society for Optics and Photonics, 2014, pp. 92340H-92340H-92345.

[23] Mao G, Li T, Zou Z, Qu S, Shi M. Prestretch effect on snap-through instability of short-length tubular elastomeric balloons under inflation. International Journal of Solids and Structures 2014;51:2109-2115.

[24] Ma J, You Z. The origami crash box. Origami 2011;5:277-290.

[25] Ma J, You Z. Energy Absorption of Thin-Walled Square Tubes With a Prefolded Origami Pattern-Part I: Geometry and Numerical Simulation. Journal of Applied Mechanics 2014;81:011003.

[26] Natori MC, Katsumata N, Yamakawa H, Sakamoto H, Kishimoto N, Conceptual model study using origami for membrane space structures, in: ASME 2013 International Design Engineering Technical Conferences and Computers and Information in Engineering Conference, American Society of Mechanical Engineers, 2013, pp. V06BT07A047V006BT007A047.

[27] Schenk M, Viquerat AD, Seffen KA, Guest SD. Review of inflatable booms for deployable space structures: Packing and rigidization. J Spacecr Rockets 2014;51:762-778.

[28] Miura K, Proposition of pseudo-cylindrical concave polyhedral shells, in: Proceedings of IASS Symposium on Folded Plates and Prismatic Structures, 1970.

[29] Hu N, Burgueño Rigoberto. Buckling-induced smart applications: recent advances and trends. Smart Materials and Structures 2015;24:063001.

[30] Albermani F, Khalilpasha $H$, Karampour H. Propagation buckling in deep sub-sea pipelines. Engineering Structures 2011;33:2547-2553.

[31] Khalilpasha H, Albermani F. Textured deep subsea pipelines. Int. J. Mech. Sci. 2013;68:224-235.

[32] Gattas JM, Wu W, You Z. Miura-Base Rigid Origami: Parameterizations of First-Level Derivative and Piecewise Geometries. Journal of Mechanical Design 2013;135:111011.

[33] Tomohiro T. Simulation of rigid origami. Origami 2009;4:175-187.

[34] Peraza-Hernandez EA, Hartl DJ, Malak Jr RJ, Lagoudas DC. Origami-inspired active structures: a synthesis and review. Smart Materials and Structures 2014;23:094001.

[35] Ahmed S, Ounaies Z, Frecker M. Investigating the performance and properties of dielectric elastomer actuators as a potential means to actuate origami structures. Smart Materials and Structures 2014;23:094003.

[36] McGough K, Ahmed S, Frecker M, Ounaies Z. Finite element analysis and validation of dielectric elastomer actuators used for active origami. Smart Materials and Structures 2014;23:094002.

[37] Martinez RV, Fish CR, Chen X, Whitesides GM. Elastomeric Origami: Programmable Paper - Elastomer Composites as Pneumatic Actuators. Adv Funct Mater 2012;22:1376-1384. 
[38] Rivlin RS. Large elastic deformations of isotropic materials. IV. Further developments of the general theory. Philosophical Transactions of the Royal Society of London A: Mathematical, Physical and Engineering Sciences 1948;241:379-397.

[39] Mooney M. A theory of large elastic deformation. J Appl Phys 1940;11:582-592.

[40] Ogden RW. Large deformation isotropic elasticity-on the correlation of theory and experiment for incompressible rubberlike solids. Proceedings of the Royal Society of London. A. Mathematical and Physical Sciences 1972;326:565-584.

[41] Fu YB, Liu JL, Francisco GS. Localized bulging in an inflated cylindrical tube of arbitrary thickness-the effect of bending stiffness. J Mech Phys Solids 2016;90:45-60.

[42] Fu YB, Xie YX. Effects of imperfections on localized bulging in inflated membrane tubes. Philosophical Transactions of the Royal Society of London A: Mathematical, Physical and Engineering Sciences 2012;370:1896-1911. 